\title{
Electron cyclotron waves in the presence of parallel electric fields in the Earth's auroral plasma
}

\author{
S. Kumar, S. K. Dixit, A. K. Gwal \\ Space Plasma Laboratory, Department of Physics, Barkatullah University, Bhopal-462 026, India
}

Received: 29 January 1996/Revised: 4 July 1996/Accepted: 8 July 1996

\begin{abstract}
The electron cyclotron waves that originate at low altitudes $\left(<0.5 \mathrm{R}_{\mathrm{E}}\right)$ and observed by ground facilities have been studied in the presence of a weak parallel electric field in auroral magnetoplasma consisting of trapped energetic auroral electrons and cold background electrons of ionospheric origin. The model distribution for auroral trapped electrons is taken as Maxwellian ring distribution. An expression for the growth rate has been obtained in the presence of parallel electric field assuming that the real frequency in the whistler mode is not affected by the presence of the electric field. The results show that waves grow (or damp) in amplitude for a parallel (or antiparallel) electric field. The influence of the electric field is more pronounced at a shorter wavelength spectrum. An increase in population of energetic electrons increases the growth rate and thus, plays a significant role in the wave excitation process in the auroral regions.
\end{abstract}

\section{Introduction}

The electric fields which were first proposed by Alfven and Fälthammar (1963) from theoretical arguments are an important parameter in the study of magnetospheric plasma and associated wave phenomena. The observations made indicate the presence of electric fields aligned parallel to the magnetic field lines in Earth's magnetoplasma (Mozer et al., 1980; Block et al., 1987; Fälthammar, 1989; Matsuoka et al., 1991; Tsuruda et al., 1994). Temerin and Mozer (1984) on the basis of measurements concluded that the average electric field in an electrostatic shock is not likely to be greater than $25 \mathrm{mV} / \mathrm{m}$. Block and Fälthammar (1990) from the Viking observations reported that weaker parallel electric fields of the order of few $\mathrm{mV} / \mathrm{m}$ to $20 \mathrm{mV} / \mathrm{m}$ are more common, with a duration that can be as long as tens of seconds. Large electric fields

Correspondence to: S. Kumar over $100 \mathrm{mV} / \mathrm{m}$ have also been reported in auroral regions (Mozer et al., 1980; Weimer and Gurnett, 1993) but they are usually confined to relatively small regions. Schriver and Ashour-Abdulla (1993) using a fully self-consistent time-dependent kinetic simulation model have shown the existence of small- and large-scale electric fields in the auroral zone. A detailed description of parallel electric fields is out of the scope of the present study, however, it is now well established that parallel electric fields occur in the auroral zone.

The ionosphere and magnetosphere are electrodynamically coupled by electric fields and the existence of electric fields therein significantly changes the electrodynamics, energization and transport of charged particles in both the regions. The wave particle interaction in the presence of electric fields may control the morphological features of whistler waves recorded at ground. Misra and Singh (1980) discussed the amplification of electromagnetic waves in an isotropic bi-Maxwellian weakly ionised plasma by electric fields in the equatorial magnetosphere. Their work is limited to small temperature anisotropy $\left(A=\left(T_{\perp} / T_{\|}-1\right)<1\right)$. Renuka and Viswanathan (1978) studied electromagnetic electron cyclotron instability in the auroral magnetosphere for higher values of temperature anisotropy. Wu et al. (1983) investigated the excitation of whistler waves in presence of auroral trapped energetic electrons $\left(n_{\mathrm{e}}\right)$ and cold background electrons $\left(n_{\mathrm{c}}\right)$ of ionospheric origin under the conditions $n_{\mathrm{e}}<n_{\mathrm{c}}$. Their work indicates that downward propagating whistler mode waves can be generated in the low altitude auroral regions at altitudes near $600 \mathrm{~km}$ by cyclotron maser instability. Wu et al. (1989) extended this study for the same region for arbitrary values of $n_{\mathrm{e}} / n_{\mathrm{c}}$.

In the present work, dealing with linear wave-particle approach, we report the effect of parallel electric field on the propagation of electron cyclotron waves in auroral magnetosphere observed by ground facilities near Fairbanks, Alaska. These observations are reported by Benson et al. (1988). The dispersion relation of Wu et al. (1989) has been modified for the presence of parallel electric field $\left(E_{\mathrm{o}}\right)$ by modifying the parallel temperature $T_{\|}$to the 
complex temperature $T_{\| \mathrm{c}}$. Under the condition $E_{\mathrm{o}}=0$ we recover the earlier known growth rate expression of $\mathrm{Wu}$ et al. (1989). We compute the growth rate for auroral magnetoplasma parameters of $\mathrm{Wu}$ et al. (1989) for various values of $E_{\mathrm{o}}=0,10,20 \mathrm{mV} / \mathrm{m}$.

\section{Theoretical considerations}

\subsection{Distribution function}

We consider a uniform, non-collisional, anisotropic, weakly ionized plasma consisting of energetic electrons and cold background electrons embedded into uniform Earth's magnetic field $\left(B_{\mathrm{o}}\right)$ and electric field parallel to $B_{\mathrm{o}}$. The energetic electrons are a portion of trapped auroral electrons which are reflected by a magnetic mirror effect at low altitudes and are again reflected back at high altitudes by the parallel electric field which occurs frequently along the auroral field lines. The drift velocity of the plasma is assumed to be much smaller than the phase velocity of waves and hence does not introduce any appreciable changes in the distribution function. We assume that ions form an immobile neutralising background. Therefore we neglect the ion dynamics completely. In such plasma the presence of a weak electric field does not lead to runaway of electrons. The model distribution function for auroral trapped electrons can be taken as Maxwellian ring distribution (Wu et al., 1989), so that:

$f\left(v_{\perp}, v_{\|}\right)=\frac{n_{\mathrm{e}} / n}{\pi^{3 / 2} \alpha_{\|} \alpha_{\perp}^{2} A} \exp \left[-\left\{\left(v_{\perp}-v_{\mathrm{o}}\right) / \alpha_{\perp}\right\}^{2}-\left(v_{\|}^{2} / \alpha_{\|}^{2}\right)\right]$

$A=\exp \left(-v_{\mathrm{o}}^{2} / \alpha_{\perp}^{2}\right)+\sqrt{\pi}\left(\frac{v_{\mathrm{o}}}{\alpha_{\perp}}\right) \operatorname{erfc}\left(-v_{\mathrm{o}} / \alpha_{\perp}\right)$,

where $\operatorname{erfc}(x)$ is the complementary error function, $n=n_{\mathrm{c}}+n_{\mathrm{e}}$ is the total electron density. The $\alpha_{\|}$and $\alpha_{\perp}$ are parallel and perpendicular electron thermal velocities and are given by:

$\alpha_{\|}=\left(\frac{k T_{\|}}{m}\right)^{1 / 2} \alpha_{\perp}=\left(\frac{k T_{\perp}}{m}\right)^{1 / 2}$.

The applied electric field parallel to $B_{\mathrm{o}}$ has an effect of modifying the electron thermal velocity in that direction from $\alpha_{\|}$to complex value $\alpha_{\| \mathrm{c}}$ given by:

$\alpha_{\| \mathrm{c}}=\alpha_{\|}\left(1-\frac{j e E_{\mathrm{o}}}{m k \alpha_{\|}^{2}}\right)^{1 / 2}$.

The $\alpha_{\| \mathrm{c}}$ in turn modifies the parallel temperature $T_{\|}$in the direction of $B_{\mathrm{o}}$ to the complex temperature $T_{\| \mathrm{c}}$ (Juhl and Treumann, 1985):

$T_{\| \mathrm{c}}=T_{\|}\left(1-\frac{j e E_{\mathrm{o}}}{k K T_{\|}}\right)$.

\subsection{Dispersion relation}

The dispersion relation for the right hand circularly polarized electromagnetic wave propagating exactly parallel to the external magnetic field (Davidson, 1983; Wu et al., 1989) in the presence of parallel electric field can be written as:

$$
\begin{aligned}
\frac{c^{2} k^{2}}{\omega^{2}}=1 & +\left(n_{\mathrm{e}} / n\right)\left(\omega_{\mathrm{e}}^{2} / \omega^{2}\right)\left[\left(\omega / k \alpha_{\| \mathrm{c}}\right) Z(\xi)\right. \\
& \left.+\left(\frac{T_{\perp}}{T_{\| \mathrm{c}}}-1\right)\{1+\xi Z(\xi)\}\right] \\
& -\left(n_{\mathrm{c}} / n\right)\left[\omega_{\mathrm{e}}^{2} / \omega\left(\omega-\Omega_{\mathrm{e}}\right)\right],
\end{aligned}
$$

where $\omega=\omega_{\mathrm{r}}+i \gamma$ is the complex frequency, $\omega_{\mathrm{e}}=$ $\left(4 \pi n e^{2} / m\right)^{1 / 2}$ is electron plasma frequency, $\Omega_{\mathrm{e}}=e B_{\mathrm{o}} / m$ is electron cyclotron frequency, and $Z(\xi)$ is the plasma dispersion function written as

$Z(\xi)=i \sqrt{\pi} \exp \left(-\xi^{2}\right)-\frac{1}{\xi}\left(1+\frac{1}{2 \xi^{2}}\right)$,

where,

$\xi=\frac{\omega-\Omega_{\mathrm{e}}}{k \alpha_{\| \mathrm{c}}}$.

The $\frac{T_{\perp}}{T_{\| \mathrm{c}}}-1=A^{\prime}$ is the modified temperature anisotropy and can be written as:

$A^{\prime}=A_{\mathrm{r}}+i A_{\mathrm{i}}$.

The parallel and perpendicular temperatures are given by

$T_{\|}=\frac{m \alpha_{\|}^{2}}{2}$

and

$T_{\perp}=\frac{m \alpha_{\perp}^{2}}{2}\left[1+\left(v_{\mathbf{0}}^{2} / \alpha_{\perp}^{2}\right)+(\sqrt{\pi} / 2 A)\left(v_{0} / \alpha_{\perp}\right) \operatorname{erfc}\left(-v_{\mathrm{o}} / \alpha_{\perp}\right)\right]$

Using Eq. (8) the dispersion relation Eq. (6) is written as: $\frac{c^{2} k^{2}}{\omega^{2}}=1+\left(n_{\mathrm{e}} / n\right)\left(\omega_{\mathrm{e}}^{2} / \omega^{2}\right)\left[\left(\omega / k \alpha_{\|}\right) Z(\xi)\right.$

$$
\begin{aligned}
& \left.+\left(A_{\mathrm{r}}+i A_{\mathrm{i}}\right)\{1+\xi Z(\xi)\}\right] \\
& -\left(n_{\mathrm{c}} / n\right)\left[\omega_{\mathrm{e}}^{2} / \omega\left(\omega-\Omega_{\mathrm{e}}\right)\right] .
\end{aligned}
$$

\subsection{Expression for growth rate}

To obtain the expression for growth rate, we solve Eq. (10) by using an asymptotic expansion for $Z(\xi)$. The effect of the electric field has been introduced only through the modification of parallel temperature.

The real frequency is not affected by the electric field (Misra and Singh, 1980; Juhl and Truemann, 1985) and only lowest order terms containing $E_{\mathrm{o}}$ are taken in the imaginary part. The real frequency is determined by cold background electrons (Wu et al., 1989),

$\frac{c^{2} k^{2}}{\omega^{2}}=1-\left(n_{\mathrm{c}} / n\right)\left[\omega_{\mathrm{e}}^{2} / \omega_{\mathrm{r}}\left(\omega_{\mathrm{r}}-\Omega_{\mathrm{e}}\right)\right]$.

If we write the dispersion equation in the form:

$D=\operatorname{Re}(D)+i \operatorname{Im}(D)$, 
then using the formula:

$\gamma=\left.\frac{-\operatorname{Im} D(\omega, k)}{(\partial / \partial \omega) \operatorname{Re} D(\omega, k)}\right|_{\omega=\omega_{\mathrm{r}}}$.

The expression for growth rate $\gamma$ is found to be:

$$
\begin{aligned}
\gamma= & \sqrt{\pi} \frac{n_{\mathrm{e}}}{n} \frac{\omega_{\mathrm{e}}^{2}}{k \alpha_{\|}} \exp \left(-\frac{\left(\Omega_{\mathrm{e}}-\omega_{\mathrm{r}}\right)^{2}}{k^{2} \alpha_{\|}^{2}}\right) \\
& \times\left[1+\frac{c^{2} k^{2}}{\omega_{\mathrm{r}}^{2}}+\frac{n_{\mathrm{c}}}{n} \frac{\omega_{\mathrm{e}}^{2}}{\left(\omega_{\mathrm{e}}-\omega_{\mathrm{r}}\right)^{2}}\right]^{-1} \\
& \times\left[\left(\frac{T_{\perp}}{T_{\|}}-1\right) \frac{\left(\Omega_{\mathrm{e}}-\omega_{\mathrm{r}}\right)}{\omega_{\mathrm{r}}}-1\right] \\
& +\frac{1}{2} \frac{n_{\mathrm{e}}}{n} \frac{e E_{\mathrm{o}}}{k K T_{\|}} \frac{\omega_{\mathrm{e}}^{2}}{\omega_{\mathrm{r}}}\left(\frac{k \alpha_{\|}}{\Omega_{\mathrm{e}}-\omega_{\mathrm{r}}}\right)^{2} \frac{\Omega_{\mathrm{e}}}{\Omega_{\mathrm{e}}-\omega_{\mathrm{r}}} .
\end{aligned}
$$

In the absence of parallel electric field i.e. $E_{\mathrm{o}}=0$ the contribution due to the last term vanishes and the expression reduces to that of the earlier known expression of $\mathrm{Wu}$ et al. (1989). A careful look at Eq. (14) reveals that in order for the instability to be of any significance, the following two conditions have to be satisfied simutaneously which determine the upper and lower bound on the unstable frequency respectively:

$\left(\frac{T_{\perp}}{T_{\|}}-1\right) \geq \frac{\omega_{\mathrm{r}}}{\Omega_{\mathrm{e}}-\omega_{\mathrm{r}}}$

$\frac{n_{\mathrm{c}}}{n} \frac{\omega_{\mathrm{e}}^{2}}{\Omega_{\mathrm{e}}^{2}} \geq \frac{c^{2}}{\alpha_{\|}^{2}} \frac{\left(\Omega_{\mathrm{e}}-\omega_{\mathrm{r}}\right)}{\omega_{\mathrm{r}}}\left[\left(1-\frac{\omega_{\mathrm{r}}}{\Omega_{\mathrm{e}}}\right)^{2}-\frac{\alpha_{\|}}{c^{2}} \frac{\omega_{\mathrm{r}}^{2}}{\Omega_{\mathrm{e}}^{2}}\right]$.

\section{Results and discussion}

The magnetosphere-ionosphere coupling has an ability in plasma to support the magnetic field aligned (parallel) electric potential drops. The Earth's magnetic field electrically and dynamically decouples to both the regions. Electric fields existing along geomagnetic field lines in the magnetospheric regions play a significant role in the wave-particle interaction. A wide variety of waves can be excited in plasma under different conditions. The study of these waves is very useful in understanding various astorphysical and geophysical phenomena and numerous plasma applications. The present study deals with linear wave-particle theory where auroral trapped energetic electrons and cold background electrons in the presence of a weak parallel electric field $\left(E_{\mathrm{o}}\right)$ interact with the right hand circularly polarized electron cyclotron waves propagating parallel to ambient magnetic field $\left(B_{\mathrm{o}}\right)$. The mean energy $\left(K T_{\|}\right)$of energetic electrons has been taken as $5 \mathrm{keV}$. Wu et al. (1983) reported the mean energy of energetic electrons to vary from 2 to $10 \mathrm{keV}$ along $B_{\mathrm{o}}$. Such energetic electrons can exist in the topside auroral ionosphere even during periods of relatively quiet ground level magnetic activity (Hardy et al., 1985).

In Figs. 1 and 2 the growth rate $\left(\gamma / \Omega_{\mathrm{e}}\right)$ has been plotted against the normalized real frequency $\left(\omega_{\mathrm{r}} / \Omega_{\mathrm{e}}\right)$ for $\omega_{\mathrm{e}} / \Omega_{\mathrm{e}}=0.1$ and 0.2 respectively for various values of $E_{\mathrm{o}}=0,10,20 \mathrm{mV} / \mathrm{m}$, when $70 \%$ of total electrons are

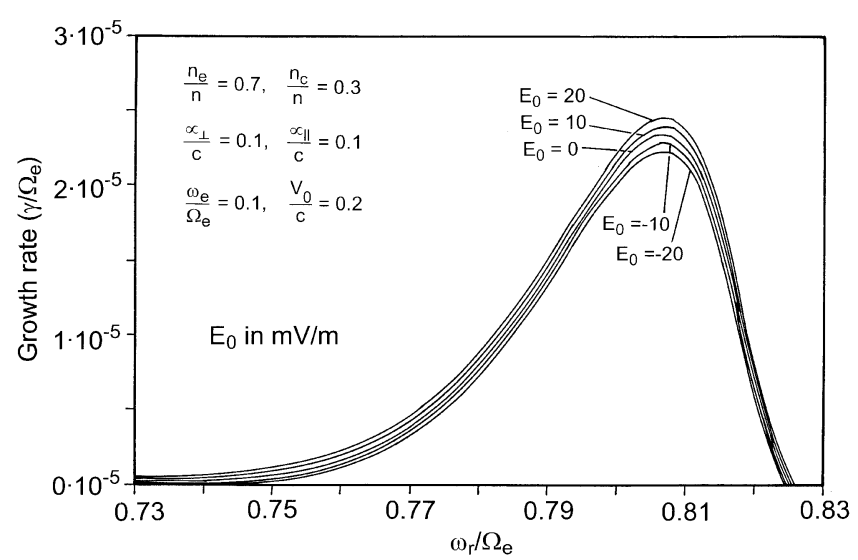

Fig. 1. Variation of growth rate $\left(\gamma / \Omega_{\mathrm{e}}\right)$ with normalised frequency $\left(\omega_{\mathrm{r}} / \Omega_{\mathrm{e}}\right)$ for $\omega_{\mathrm{e}} / \Omega_{\mathrm{e}}=0.1, \alpha_{\|} / c=\alpha_{\perp} / c=0.1, v_{\mathrm{o}} / c=0.2, n_{\mathrm{e}} / n=0.7$, and for different values of $E_{\mathrm{o}}=0,10,20, \mathrm{mV} / \mathrm{m}$

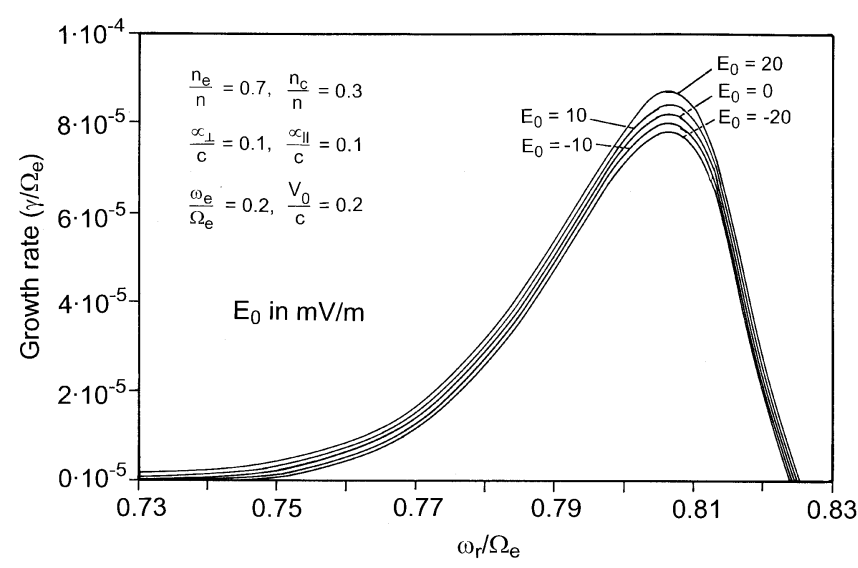

Fig. 2. Variation of growth rate $\left(\gamma / \Omega_{\mathrm{e}}\right)$ with normalised frequency $\left(\omega_{\mathrm{r}} / \Omega_{\mathrm{e}}\right)$ for $\omega_{\mathrm{e}} / \Omega_{\mathrm{e}}=0.2, \alpha_{\|} / c=\alpha_{\perp} / c=0.1, v_{\mathrm{o}} / c=0.2, n_{\mathrm{e}} / n=0.7$, and for different values of $E_{o}=0,10,20 \mathrm{mV} / \mathrm{m}$

energetic (i.e. $n_{\mathrm{e}} / n=0.7$ ). The trapped energetic electron parameters are taken as $\alpha_{\|} / c=\alpha_{\perp} / c=0.1, v_{\mathrm{o}} / c=0.1$. From these figures we can see that as the $\omega_{\mathrm{r}} / \Omega_{\mathrm{e}}$ increases the growth rate increases slowly but falls very rapidly from its maximum value at around $\omega_{\mathrm{r}} / \Omega_{\mathrm{e}}=0.8$. The electric field parallel (or antiparallel) to the wave vector enhances (or reduces) the growth rate. The effect of the electric field is more pronounced towards the larger values of $\omega_{\mathrm{r}} / \Omega_{\mathrm{e}}$. In the absence of an electric field i.e. $E_{\mathrm{o}}=$ $0 \mathrm{mV} / \mathrm{m}$ we recover the results of $\mathrm{Wu}$ et al. (1989). The frequency of the domain i.e. the range of $\omega_{\mathrm{r}} / \Omega_{\mathrm{e}}$ in which instability exists is also increased (or decreased) as the applied parallel (or antiparallel) electric field increases. This increase in frequency domain under the parallel electric field is less compared to that reported by Misra and Singh (1980) for whistler mode instability in the equatorial region of the Earth's magnetosphere. In the presence of parallel electric field the electron distribution becomes loss-cone distribution (Juhl and Treumann, 1985) and the parallel electric field changes the resonance condition, hence the growth/damping of the wave is expected. Wave growth requires a source of free energy in 
plasma. The increase in wave growth in the presence of parallel electric field is due to the acceleration of electrons in the electric field so that they get into resonance with the waves and transfer free energy to the wave leading wave growth. However, in the case of an antiparallel electric field the decrease in growth is due to deceleration of electrons in the electric field as a result of which they escape from resonance with the wave. A detailed study on the acceleration of electrons in presence of a parallel electric field has been carried by Kaufmann et al. (1976) and Block and Fälthammar (1990). Fig. 3 shows the variation of $\gamma / \Omega_{\mathrm{e}}$ with temperature anisotropy parameter $A\left(T_{\perp} / T_{\|}-1\right)$ for different values of $E_{\mathrm{o}}=0,10,20 \mathrm{mV} / \mathrm{m}$. It is evident from Fig. 3 that a minimum value of temperature anisotropy is necessary for the excitation of electron cyclotron waves whose dispersion relation belongs to the same branch as whistler waves. It may now be inferred that temperature anisotropy is a main source of the free energy to electron cyclotron waves and the parallel electric field further amplifies these waves.

The variation of $\gamma / \Omega_{\mathrm{e}}$ against the $\omega_{\mathrm{e}} / \Omega_{\mathrm{e}}$ has been shown in Fig. 4 for $\alpha_{\|} / c=\alpha_{\perp} / c=0.1, v_{\mathrm{o}} / c=0.2, \omega_{\mathrm{r}} / \Omega_{\mathrm{e}}=0.80$. The effect of electric field increases with increase in $\omega_{\mathrm{e}} / \Omega_{\mathrm{e}}$. From Fig. 4 it may be noted that such an instability can also occur in the regions where plasma frequency is less than the electron cyclotron frequency, provided that energetic electron density is large compared to the cold electron density. Finally in Fig. 5, we present the effect of added energetic electrons on the wave growth. Shown in Fig. 5 are the plots of $\gamma / \Omega_{\mathrm{e}}$ against density ratio of energetic to cold electrons $\left(n_{\mathrm{e}} / n_{\mathrm{c}}\right)$ for $\alpha_{\|} / c=\alpha_{\perp} / c=0.1, v_{\mathrm{o}} / c=$ $0.2, \omega_{\mathrm{r}} / \Omega_{\mathrm{e}}=0.80, \omega_{\mathrm{e}} / \Omega_{\mathrm{e}}=0.2$ and for various values of $E_{\mathrm{o}}=0,10,20 \mathrm{mV} / \mathrm{m}$. It is clearly seen from Fig. 5 that an added population of auroral trapped energetic electrons increases the growth of waves. The background electrons of ionospheric origin also play a significant role in the emission processes. Wu et al. (1989) pointed out that if the density of cold background electrons prevails over that of energetic electrons no emission of electron cyclotron waves is possible when the plasma frequency is less than

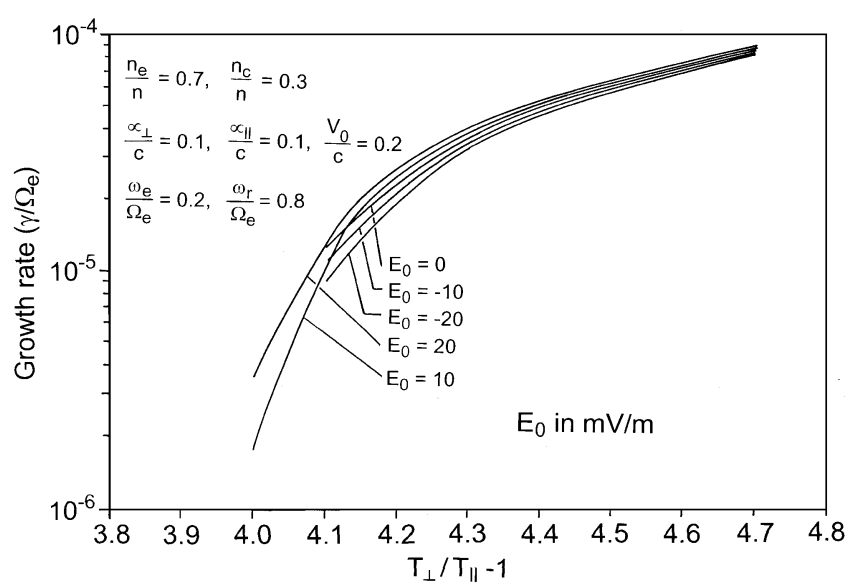

Fig. 3. Variation of growth rate $\left(\gamma / \Omega_{\mathrm{e}}\right)$ with $\left(T_{\perp} / T_{\|}-1\right)$ for $\omega_{\mathrm{e}} / \Omega_{\mathrm{e}}=0.2, \alpha_{\|} / c=\alpha_{\perp} / c=0.1, v_{\mathrm{o}} / c=0.2, n_{\mathrm{e}} / n=0.7$, and for different values of $E_{\mathrm{o}}=0,10,20 \mathrm{mV} / \mathrm{m}$

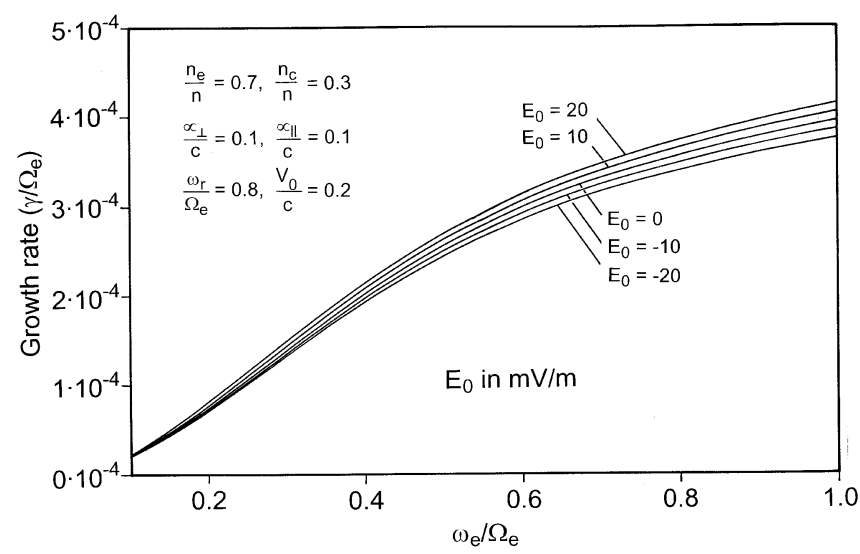

Fig. 4. Variation of growth rate $\left(\gamma / \Omega_{\mathrm{e}}\right)$ with $\omega_{\mathrm{e}} / \Omega_{\mathrm{e}}$ for $\alpha_{\|} / c=$ $\alpha_{\perp} / c=0.1, v_{\mathrm{o}} / c=0.2, n_{\mathrm{e}} / n=0.7, \omega_{\mathrm{r}} / \Omega_{\mathrm{e}}=0.8$ and for different values of $E_{\mathrm{o}}=0,10,20 \mathrm{mV} / \mathrm{m}$

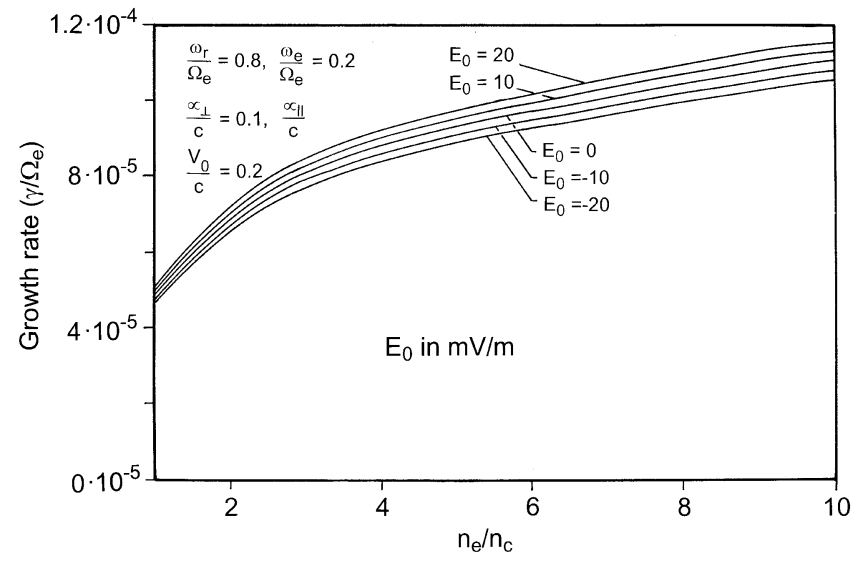

Fig. 5. Variation of growth rate $\left(\gamma / \Omega_{\mathrm{e}}\right)$ with $n_{\mathrm{e}} / n_{\mathrm{c}}$ for $\alpha_{\|} / c=$ $\alpha_{\perp} / c=0.1, v_{\mathrm{o}} / c=0.2, \omega_{\mathrm{e}} / \Omega_{\mathrm{e}}=0.2$, and for different values of $E_{\mathrm{o}}=0$, $10,20 \mathrm{mV} / \mathrm{m}$

the gyrofrequency. Benson and Wong (1987) pointed out that whistler mode signals observed in the topside of the ionosphere can be due to either the Cerenkov resonance (Maggs, 1976) or the cyclotron resonance (Wu et al., 1983) but only the latter allows the propagation of waves parallel to ambient magnetic field that reach the ground facilities (Wu et al., 1989). Benson et al. (1988) presented evidence in support of the suggestion of Benson and Wong (1987) that the cyclotron maser generation of downward propagating whilster mode waves in the topside ionosphere can be detected at ground level in the auroral region. Of particular significance here is a means to explain the increase in growth rate for an added population of energetic electrons and the effect of the parallel electric field. The electric field parallel to the terrestrial magnetic field accelerates the electrons towards the Earth in regions of auroral arc (Hoffman and Evans, 1968). These electrons carry a substantial amount of free energy to excite the instability. Prasad and Singh (1981) have reported that the D.C. electric field plays an important role and may work in simultaneity with other processes for enhancement of electron precipitation. The plasma sheet is 
relatively energetic compared to the ionosphere and provides a source of high energy $(T \sim \mathrm{keV})$ plasma that flows from high altitudes to the auroral zone. The parallel electric field therein further causes the electrons to drift into the auroral zone increasing the energetic electron density which provides a source of free energy and the growth of electron cyclotron waves.

Acknowledgements. The authors (SK and SKD) are grateful to the Council of Scientific and Industrial Research (CSIR), New Delhi for financial support.

Topical Editor K.-H. Glaßmeir thanks a referee for his help in evaluating this paper.

\section{References}

Alfven, H., and C. G. Fälthammar, Cosmical Electrodynamics, Clarendon, Oxford, 1963.

Benson, R. F., and H. K. Wong, Low-altitude ISIS-I observations of auroral radio emissions and their significance to the cyclotron maser instability, J. Geophys. Res., 92, 1218-1230, 1987.

Benson, R. F., M. D. Desch, R. D. Hunsucker, and G. J. Romick, Ground-level detection of low- and medium-frequency auroral radio emissions, J. Geophys. Res., 93, 277-283, 1988.

Block, L. P., and C.-G. Fälthammar, The role of magnetic-fieldaligned electric fields in auroral acceleration, J. Geophys. Res., 95, 5877-5888, 1990.

Block, L. P., C.-G. Fälthammar, P.-A. Lindqvist, G. T. Marklund, F. S. Mozer and A. Pedersen, Electric field measurements on Viking: first results, Geophys. Res. Lett., 14, 435-438, 1987.

Davidson, R. C., Kinetic waves and instabilities in uniform plasma, Handbook of Plasma Physics, Eds. A. A. Galeev and R. N. Sudan, North Holland, Amsterdam, vol. 1, pp. 521-585, 1983.

Fälthammar, C. G., Electric fields in the magnetosphere; the evidence from ISEE, GEOS and Viking, IEEE Trans. Plasma Sci., 17, 174-181, 1989.

Hoffman, R. A., and D. S. Evans, Field-aligned electron bursts at high latitude observed by Ogo 4, J. Geophys. Res., 73, 6201-6214, 1968.

Hardy, D. A., M. S. Gussenhoven, and E. Holeman, A statistical model of auroral electron precipitation, J. Geophys. Res., 90, 4229-4248, 1985.
Juhl, B., and R. A. Treumann, VLF emission stimulated by parallel electric fields, J. Plasma Phys., 34, 47-66, 1985.

Kaufmann, R. L., D. N. Walker, and R. L. Arnoldy, Acceleration of auroral electrons in parallel electric field, J. Geophys. Res., 81, 1673-1678, 1976.

Maggs, J. E., Coherent generation of VLF hiss, J. Geophys. Res., 81, 1707-1724, 1976.

Matsuoka, A., T. Mukai, H. Hayakawa, Y. I. Kohno, K. Tsuruda, A. Nishida, T. Okada, N. Kaya, and H. Fukunishi, EXOS-D observations of electric field fluctuations and charged particle precipitation in the polar cusp, Geophys. Res. Lett., 18, 305-308, 1991.

Misra, K. D., and B. D. Singh, On the modifications of the whistler mode instability in the magnetosphere in the presence of parallel electric field by cold plasma injection, J. Geophys. Res., 85, 5138-5142, 1980.

Mozer, F. S., C. A. Cattell, M. K. Hudson, R. L. Lysak, M. Temerin, and R. B. Torbert, Satellite measurements and theories of low altitude auroral particle acceleration, Space Sci. Rev., 27, 155-213, 1980.

Prasad, R., and R. N. Singh, Effect of parallel electric field on charged particle precipitation, Planet. Space Sci., 29, 915-924, 1981.

Renuka, G., and K. S. Viswanathan, Instabilities of the whistler mode in the magnetosphere, Indian J. Radio Space Phys., 7, 248-253, 1978.

Schriver, D., and M. Ashour-Abdalla, Self-consistent formation of parallel electric fields in the auroral zone, Geophys. Res. Lett., 20, 475-478, 1993.

Temerin, M., and F. S. Mozer, Observations of the electric fields that accelerate auroral particles, Proc. Indian Acad. Sci. (Earth Planet. Sci.), 93, 227-245, 1984.

Tsuruda, K., H. Hayakawa, M. Nakamura, T. Okada, A. Matsuoka, F. S. Mozer, and R. Schmidt, Electric field measurements on the GEOTAIL satellite, J. Geomag. Geoelectr., 46, 693-711, 1994.

Weimer, D. R., and D. A. Gurnett, Large amplitude auroral electric fields measured with DE 1, J. Geophys. Res., 98, 13,557-13,564, 1993.

Wu, C. S., D. Dillenburg, L. F. Ziebell, and H. P. Freund, Excitation of whistler waves by reflected auroral electrons, Planet. Space Sci., 31, 499-507, 1983.

Wu, C. S., P. H. Yoon, and H. P. Freund, A theory of electron cyclotron waves generated along auroral field lines observed by ground facilities, Geophys. Res. Lett., 16, 1461-1464, 1989. 